ACTA SCIENTIFIC PHARMACEUTICAL SCIENCES (ISSN: 2581-5423)

Volume 3 Issue 7 July 2019

Short Communication

\title{
Pharmacogenetics in Clinical Practice
}

\section{Mohamed EE Shams*}

Professor, Clinical Pharmacy Practice, Faculty of Pharmacy, University of Mansoura, Egypt

*Corresponding Author: Mohamed EE Shams, Professor, Clinical Pharmacy Practice, Faculty of Pharmacy, University of Mansoura, Egypt.

Received: May 20, 2019; Published: June 07, 2019

DOI: $10.31080 /$ ASPS.2019.03.0306

Nowadays, pharmaceutical companies are limited to developing drugs using a "one size fits all" system. However not all patients respond with the same manner to drugs and many of them experience adverse drug reactions and even deaths while failure of treatment may be found in the others. More than 20 -fold inter-individual variabilities in the steady state concentrations of the drugs inside the body may result upon administration the same dose. This may be due to patients differ in their ability to absorb, distribute, metabolize and excrete drugs due to concurrent disease(s), age, concomitant medication(s) or genetic peculiarities. Pharmacogenetics as a term comes from a combination of two words: pharmacology and genetics. Therefore, pharmacogenetics is the study of genetic factors and how an individual's genetic inheritance can affects the body's response to drugs.

Common variations in the genetic information include changes to a single letter of the four nucleotide bases of the DNA code - A, T, C and G. For example, the DNA letter ' $C$ ' may exhibit mutation to 'A' or even deleted so that the message from the gene has been slightly changed. This is known as single nucleotide polymorphism (SNP). In most cases, these variations due to SNPs usually produce no obvious effect or direct health problem. However, in some patients it can affect their response to a drug. For example, the effects of drugs like angiotensin converting enzyme inhibitors (ACE inhibitors) that improve symptoms and survival in patients with congestive heart failure have been found to be greater in people of European ancestry than African-Americans. Genetic screening of patients prior to treatment will eventually enable this knowledge to be applied in clinical practice.

In addition, some drugs act by binding to receptor sites, on the surface or within the body cells. Variation in the genes that code for these receptors means that some patients may produce receptors that do not interact properly with the drug. For example, some patients have a lack of response to the bronchodilator drug "salbutamol", which is used in treatment of asthma. This may be due to a genetic polymorphism in the gene that codes a receptor on the surface of smooth muscle cells lining the airways of the lungs.

\section{Drug targets}

Genetic polymorphisms are commonly happened in drug target proteins, including receptors, enzymes, ion channels, and intracellular signaling proteins. Drug target genes may produce its effect in concert with the genes that affect pharmacokinetic properties (i.e., genes for drug transporters and drug-metabolizing enzymes) to contribute to overall drug response. For example, the genes for CYP2C9, which is the major metabolizing enzyme for S-warfarin, and vitamin $\mathrm{K}$ oxidoreductase (VKOR), the target enzyme for warfarin, interact to affect warfarin dose response.

Genes may also determine how many of the receptors are produced on or within cells. Genetic polymorphism in this case means that some people may produce more of these sites than others. For example, a research study has shown that women with metastatic breast cancer (cancer that can spread to other organs) who overexpress the protein product of the gene called HER2 have aggressive disease and a poor prognosis. The HER2 gene normally produces a receptor protein on the surface of the breast cells that is thought to play a role in their normal cell growth by signaling the cell to divide and multiply. When the HER2 gene is over-expressed, extra protein receptors are produced on the cell surface. This appears to trigger the cell to grow and divide out of control, and the cell becomes cancerous. Twenty to thirty percent of all women with metastatic breast cancer over-express the HER2 protein. The drug Herceptin $^{\circledR}$ is an artificially developed antibody against the HER2 gene product and is called a monoclonal antibody. It is thought that Herceptin ${ }^{\circledR}$ works by binding to the receptor sites on the cell sur- 
face, thereby limiting the amount of cell division that occurs and preventing the growth of the cancer.

\section{Drug metabolism}

The way at which people absorb, distribute, metabolize and excrete drugs in the body can also be impacted upon by their genetic information. The liver enzymes cytochrome $\mathrm{P} 450$ are responsible for metabolizing more than 30 different classes of drugs. DNA variations in genes that code for these enzymes can affect their ability to metabolize certain drugs. Patients who are considered as "poor metabolizers" of these enzymes are unable to break down and eliminate drugs from the body can cause drug overdose and adverse drug reactions. For example, codeine as a pain killer is metabolized by a protein (an enzyme) produced in the liver called CYP2D6. People who are poor metabolizers and have low levels of this enzyme metabolize codeine slowly and so it will be in the body for a longer period of time. Consequently, poor metabolizers of codeine are more prone to have respiratory side effects than normal extensive metabolizers. Those people will require smaller doses of the drugs that are metabolized by this enzyme, while ultra-extensive metabolizers due to gene duplication of multiplication and have a lot of the enzyme, will need mega doses of the drug to get the same effects.

Recently, researchers use genetic tests for variations in cytochrome P450 genes to screen and monitor patients. In addition, many pharmaceutical companies screen their chemical compounds to see how well they are broken down by variant forms of CYP enzymes.

This means instead of the standard trial-and-error method of matching patients with the right drugs, physicians will be able to analyze a patient's genetic profile and prescribe the best available drug therapy from the beginning. This will speed recovery time and increase safety as well as the likelihood of adverse reactions will be eliminated.

\section{Drug development}

By excluding from clinical trials those people whose genetic makeup would make the drug being tested harmful or ineffective for them will increase the chance that a drug will show itself useful to a particular population group. This would increase the chance that the same drug will make it into the marketplace. For example, what was shown in clinical trials for developing drugs for Alzheim- er disease and other forms of dementia. The first gene to be identified that is associated with Alzheimer disease in later life is called APOE. The APOE gene occurs in three forms known as E2, E3 and E4. Having the APOE-4 form of the gene has been shown to be associated with Alzheimer disease. APOE-4 is also distinctly involved in drug treatment for Alzheimer disease. Individuals with Alzheimer disease who have E2 and E3 forms of the APOE genes respond well to the drug Tacrine ${ }^{\circledR}$, while those with APOE-4 do not. Knowledge of the APOE genetic make-up of an individual with Alzheimer disease is important in drug treatment trials.

In conclusion, the implementation of pharmacogenetic testing in clinical practice will cause substantial reduction in the expenses involved in drug development, ensure a safe clinical trial and reduce failures of treatment. In other words, pharmacogenetics testing can improve health and save lives of people around the world.

\section{Volume 3 Issue 7 July 2019 \\ (C) All rights are reserved by Mohamed EE Shams.}

